\title{
Telemedizin als Bestandteil der
}

\section{GKV-Regelversorgung}

\section{Bestandsaufnahme und Regelungsvorschläge für eine arztzentrierte Versorgung}

\section{STEFFEN BOHM, FRANZ KNIEPS, AXEL WEHMEIER}

Steffen Bohm ist Geschäftsführer der AGENON Gesellschaft für Unternehmensentwicklung im Gesundheitswesen $\mathrm{mbH}$ in Berlin

Dr. Axel Wehmeier ist Leiter des Konzerngeschäftsfeld Gesundheit der Deutschen Telekom

Franz Knieps ist Vorstandsvorsitzender des BKK Dachverband e.V. in Berlin und Herausgeber der Zeitschrift „Gesundheits- und Sozialpolitik“

\author{
Erfahrungen in anderen Ländern und Projekte \\ hierzulande zeigen, dass telemedizinische Lösungen \\ eine Vielzahl von Möglichkeiten bieten, die medizinische \\ Versorgung zu unterstützen. Im Koalitionsvertrag ist \\ formuliert, dass telemedizinische Leistungen gefördert \\ und angemessen vergütet werden sollen. Im folgenden \\ Beitrag werden für die vertragsärztliche Versorgung \\ telemedizinischen Anwendungsfelder identifiziert \\ und in eine Ordnung gebracht, die für die Umsetzung \\ in eine EBM-Vergütungsregelung in Frage kommen.
}

\section{Einleitung}

Die vielfältigen Möglichkeiten, die ambulante ärztliche medizinische Versorgung durch den Einsatz telemedizinischer Lösungen zu unterstützen, sind bislang nicht im Regelversorgungssystem angekommen. Neben berufsrechtlichen und datenschutzrechtlichen Fragen steht dem auch entgegen, dass die Möglichkeiten, ambulante ärztliche Leistungen entsprechend unterstützt zu erbringen, im bundesweit einheitlich gültigen Leistungs- und Vergütungskatalog für die vertragsärztliche Versorgung - dem Einheitlichen Bewertungsmaßstab für ärztliche Leistungen (EBM) - bislang nur in zwei eng eingegrenzten Anwendungsfällen explizit berücksichtigt sind. Das GKVVersorgungsstrukturgesetz (GKV-VStG) beinhaltet Vorgaben, nach denen eine Anpassung des EBM im Hinblick auf die Vergütung telemedizinisch unterstützter vertragsärztlicher Leistungserbringung zu prüfen und vorzunehmen ist. Die konkrete Umsetzung steht bislang noch aus. Im Koalitionsvertrag der Regierungsparteien findet sich aktuell die Formulierung: „Te- lemedizinische Leistungen sollen gefördert und angemessen vergütet werden." ${ }^{\text {"Nach- }}$ folgend wird die Diskussion um Telemedizin in den versorgungspolitischen Kontext eingebettet, es werden definitorische und organisatorische Aspekte beleuchtet, und es wird ein konkreter Vorschlag unterbreitet, wie der gesetzliche Auftrag umgesetzt werden kann.

\section{Telemedizin im Kontext der versorgungspolitischen Diskussion}

Die gesundheitliche Versorgung in Deutschland ist gekennzeichnet durch ein vergleichsweise hohes Leistungsund Qualitätsniveau ohne wesentliche Zugangsbarrieren. Die Akzeptanz von Versicherung und Versorgung in der Bevölkerung ist hoch, auch wenn internationale Vergleiche und nationale Analysen dem Gesundheitssystem Schwachstellen und Veränderungsresistenz nachweisen. Speziell die Herausforderungen der sozio-

1 Deutschlands Zukunft gestalten. Koalitionsvertrag zwischen CDU, CSU und SPD. 18. Legislaturperiode, S. 77. 
demografischen und der medizinisch-technischen Entwicklung werden durch die traditionellen Akteure, die Steuerungsverantwortung im Gesundheitswesen tragen, nur unzureichend beantwortet.

Exemplarisch für strukturelle Mängel im Gesundheitssystem stehen die historisch überholte und ineffiziente Zweiteilung des Versicherungsmarktes, die Leistungserbringung in voneinander weitgehend abgeschotteten Sektoren, die stetig fortschreitende Spezialisierung zulasten der flächendeckenden Grundversorgung, die Konzentration der (fachärztlichen) Versorgung in Städten und die geringe Innovationsdiffusion und Outcome-Orientierung im Vergleich zu anderen Branchen.

Die hier nur skizzierten Herausforderungen und Mängel können vor allem über kooperative Organisations- und Betriebsformen, indikations- und populationsorientierte integrierte Versorgungskonzepte, arztentlastende Delegation und Substitution sowie durch neue Mobilitätskonzepte und Technologien gemeistert werden. Nur ein Zusammenwirken von unterschiedlichen politischen Steuerungsansätzen wird Behandlungsprozesse optimieren und die flächendeckende Versorgung auch in der Zukunft gewährleisten. Dies gilt im besonderen Maße für die ambulante ärztliche Versorgung, die in Deutschland weitgehend von selbstständigen niedergelassenen Vertragsärzten in Einzelpraxen erbracht wird, auch wenn der Trend zu kooperativen Praxisformen (Praxisgemeinschaften, Gemeinschaftspraxen, Ärztenetze und -verbünde, Medizinische Versorgungszentren) stetig zunimmt. Anders als in vielen anderen Industrieländern ist die fachärztliche Versorgung nicht am Krankenhaus konzentriert, obwohl in der spezialfachärztlichen Versorgung eine zunehmende Konkurrenz zwischen niedergelassenen Vertragsärzten und Krankenhäusern entstanden ist und vom Gesetzgeber in der Neufassung des $\mathbb{1 1 6}$ b SGB V ausdrücklich als eigenständiger Versorgungsbereich weitgehend regulierungsfrei geregelt ist.

In den letzten Jahren wurden in verschiedenen Bereichen und verschiedenen Regionen unterschiedliche telemedizinische Ansätze in der gesundheitlichen Versorgung entwickelt und implementiert. Dabei stehen die folgenden Dimensionen dieser Ansätze zur Diskussion:

- medizinische Inhalte, die für telemedizinische Anwendungen infrage kommen;
- technische Möglichkeiten, die telemedizinische Ansätze bieten;

- betriebswirtschaftliche Voraussetzungen für Investitionen in und Vergütung von telemedizinischen Anwendungen;

- Einbettung telemedizinischer Anwendungen in das Leistungs-, Vertrags- und Organisationsgefüge des Gesundheitswesens (und ggf. Anpassung dieses Gefüges).

Telemedizinische Anwendungen in Diagnostik und Therapie sind hier sowohl in den Beziehungen zwischen Arzt und Patient als auch in den Beziehungen zwischen Ärzten möglich. Dabei können andere Gesundheitsberufe - wie zum Beispiel Krankenschwestern oder medizinische Fachangestellte - einbezogen werden.

Nachdem bereits $\mathbb{} 67$ SGB V vorgibt, dass „die papiergebundene Kommunikation so bald und so umfassend wie möglich durch die elektronische und maschinell verwertbare Übermittlung von Befunden, Diagnosen, Therapieempfehlungen, und Behandlungsberichten" ersetzt werden soll, hat das GKV-VStG die Rahmenbedingungen für den Einsatz von telemedizinischer Lösungen substanziell verbessert: Gemäß \87 Abs. 2a Satz 8 SGB V sollte der Bewertungsausschuss (BA) bis spätestens zum 31.10.2012 prüfen, in welchem Umfang telemedizinische Leistungen im Bereich der über den EBM bundesweit einheitlich geregelten vertragsärztlichen Versorgung und ihrer Vergütung erbracht werden können. In der Gesetzesbegründung zum GKV-VStG ist klargestellt, dass sich die Prüfung auch auf den Anpassungsbedarf der bundesmantelvertraglich vereinbarten Kostenpauschalen (Kapitel 40 des EBM) hinsichtlich der elektronischen Übermittlung ärztlicher Unterlagen zu erstrecken hat. Bis zum 31.03.2012 sollte der BA auf dieser Grundlage beschließen, inwieweit der EBM anzupassen ist. Bei einer Anpassung sollen für telemedizinisch erbrachte Leistungen Einzelleistungen oder Leistungskomplexe vorgesehen werden ( $\int 87$ Abs. 2b Satz 1 SGB V).

Der damit vom Gesetzgeber vorgegebene Zeitplan konnte nicht eingehalten werden. Die maßgeblichen Akteure auf Bundesebene - Kassenärztliche Bundesvereinigung (KBV), Spitzenverband Bund der gesetzlichen Krankenversicherung (GKV-Spitzenverband) unter enger Abstimmung mit den Bundesorganisationen der gesetzlichen Krankenkassen - sind in den relevanten Gremien aktuell noch mit der Umsetzung des gesetzlichen Auftrages befasst. Daher halten es die Autoren für zielführend, einen eigenen Vorschlag zur Umsetzung der gesetzlichen Vorgaben zu machen, um den Diskussions- und Entscheidungsprozess zu beleben.

\section{Telemedizin als Bestandteil der Regelversorgung: Definitorische und organisatorische Aspekte}

Charakteristisch für die derzeit unter dem Begriff „Telemedizin“ praktizierten Versorgungsansätze ist, dass sie zwar einheitlich das Merkmal aufweisen, medizinische Versorgungsprozesse durch die Möglichkeiten der Übermittlung von Informationen via Datenfernübertragung (DFÜ) und ihrer maschinellen Weiterverarbeitung zu unterstützen. Ausgehend von diesem gemeinsamen Merkmal weisen die praktizierten Lösungen dann aber eine große Heterogenität auf, die es mitunter schwer macht, ein klares begriffliches und inhaltliches Verständnis von „Telemedizin“ zu entwickeln ${ }^{2}$.

Übersicht 1 zeigt, welche unterschiedlichen Ansätze unter dem Oberbegriff „Telemedizin“ aktuell in der Praxis umgesetzt und diskutiert werden.

Die Umsetzung der verschiedenen Ansätze erfolgt in Deutschland bisher in oft sehr kleinen Pilotprojekten. Schwerpunkte bilden hier vor allem die unter Begriffen wie Telemonitoring, Telediabetes, Telekardiologie, Telepsychiatrie geführten Ansätze, in denen die Beobachtung via DFÜ übermittelter Vitalparameter und gegebenenfalls die Anpassung der Therapie oder auch die Rund-um-die-UhrÜberwachung von Vitalparametern bei Hochrisikopatienten im Mittelpunkt steht.

Bei der Beobachtung übermittelter Vitalparameter zur Therapieanpassung bzw. -sicherung steht ganz analog etwa zu einem Langzeit-EKG oder einem DiabetesTagebuch im Rahmen der konventionellen Behandlung - die Erhebung und Sichtung von medizinisch relevanten Daten im Vordergrund. Die Vitaldaten des Patienten werden dabei über ein mobiles Medizingerät erfasst, welches die Daten in eine Patientenakte direkt über eine gesicherte

2 Vgl. dazu z.B. Schräder WF, B Lehmann, F Hezel, R Beckers (2009): Entwicklung der Telemedizin im Land Brandenburg aus versorgungsinhaltlicher Sicht, Gutachten im Auftrag des Gesundheitsministeriums im Land Brandenburg, http://www.masf. brandenburg.de/media/lbm1.a.1336.de/ telemedizin.pdf, S. $18 \mathrm{ff}$. 
Datenverbindung einspeisen kann. Dabei werden die Daten nicht 1:1 weitergegeben, sondern werden mittels entsprechender Software aggregiert, vorausgewertet und gegebenenfalls vorinterpretiert, so wie es etwa bei Labordaten bereits üblich ist. Bei schwer erkrankten Patienten mit stark risikobehaftetem Verlauf kommt über die bloße Erfassung und Aufbereitung der Vitaldaten noch die Notwendigkeit hinzu, die Versorgung des Patienten in Notfällen unverzüglich sicherzustellen. Dies ist beispielsweise das Design der sogenannten Fontane-Studie, in der HerzinsuffizienzPatienten der Stufen NYHA III und IV rund um die Uhr von dem an der Charité angesiedelten Telemedizinischen Zentrum (TMZ) überwacht werden.

Charakteristisch ist bislang, dass in diesen Projekten der versorgungspolitische Impuls und auch die Steuerung durch die Krankenkassen in selektivvertraglich geregelter Zusammenarbeit mit Kliniken und Managementgesellschaften erfolgt ${ }^{3}$. Oder aber es sind Kliniken, wie die Charité im genannten Beispiel (Projekt „Partnership for the Heart“), die über öffentliche Fördermittel die Finanzierung ihrer telemedizinischen Entwicklungen sicherstellen. Die niedergelassenen Ärzte schreiben sich dabei gegen ein zusätzliches Entgelt zumeist „nur“ in die gesondert aufgesetzten Programme der Krankenkassen ein oder nehmen an den Förderprojekten mit ihren Patienten teil. Sie erhalten dann von der Managementgesellschaft oder der Klinik regelmäßig zusammenfassende Berichte, ohne aber in der Regel aktiv an dem telemedizinisch gestützten Versorgungsprozess teilzuhaben.

\section{Übersicht 1}

„Tele-“ Kurzbezeichnung

Telekommunikation

Telekonsultation

Telekonsil, Teleradiologie, Teleophthalmologie

Teleonkologie

Telemonitoring, Telediabetes, Telekardiologie, Telepsychiatrie

Telepathologie

Telechirurgie

Quelle: AGENON Berlin
Diese Konstellation würde sich bei einer Berücksichtigung über entsprechende Gebührenordnungsziffern (GOPs) im EBM grundlegend verändern. Werden Ansätze, wie die zuvor beschriebenen, mit der Aufnahme in den EBM integraler Bestandteil der GKV-Regelversorgung, so würde nunmehr der Vertragsarzt die Entscheidung treffen, ob er die Behandlung telemedizinisch unterstützt erbringt und soweit erforderlich - entsprechende Veranlassungen vornimmt oder ob er vollständig im Rahmen der konventionellen Leistungserbringung verbleibt.

Unter organisatorischen Gesichtspunkten ist noch ein zweiter Aspekt von Relevanz: Soweit die telemedizinisch unterstützte ärztliche Versorgung voraussetzt, dass Vitaldaten des Patienten erhoben, übertragen, gesichtet und bewertet werden, müssen die bislang in Projekten praktizierten Verfahren zwingend weiterentwickelt und harmonisiert werden. In den Projekten werden erforderliche Patientenendgeräte in der Regel durch die TelemedizinProvider direkt auf Veranlassung des TMZ bzw. der forschenden Klinik zum teilnehmenden Patienten gebracht und entweder direkt von der Krankenkasse (im Fall eines Selektivvertrages) oder aus Fördermitteln finanziert. Für die Regelversorgung wird dieses Verfahren anders aufgesetzt werden müssen, weil in diesem Fall zum einen der Arzt die telemedizinische Betreuung initiiert und zum anderen eine GKV-weit einheitliche Lösung für die Finanzierung der Endgeräte erforderlich ist (siehe dazu auch unten Abschnitt 4.6).
Drittens schließlich hat eine Anpassung des EBM an die Möglichkeiten der Telemedizin eine technische Dimension: Die telemedizinisch unterstützte ambulante ärztliche Versorgung wird sich als Bestandteil der Versorgungsroutinen in der Fläche nur soweit verbreiten können, wie das Problem des Imports von Daten in die Praxisverwaltungssysteme (PVS) und ihre Weiterverarbeitung gelöst werden kann.

All diese Punkte sprechen dafür, nach konkreten Anhaltspunkten für die Berücksichtigung von Telemedizin im EBM zu suchen.

\section{Ansatzpunkte für die Berücksichtigung von Telemedizin im EBM}

Historisch ist der EBM Ende der siebziger Jahre des letzten Jahrhunderts aus den verschiedenen kassenartenspezifischen Gebührenordnungen hervorgegangen. Seitdem ist er in einer Reihe von Reformen überarbeitet und angepasst worden, zuletzt mit einer „großen“ EBM-Reform zum 1. Januar 2008.

Bundeseinheitlich werden die vertragsärztlichen Leistungen und ihre Bewertung im EBM aktuell in 34 Kapiteln und knapp 2.300 Positionen, den sogenannten Gebührenordnungspositionen (GOPs), geregelt. Hinzu kommt ein weiteres Kapitel, in dem in insgesamt 104 Positionen die sogenannten Sachkostenpauschalen geregelt sind (Kapitel 40 des EBM).

Die Entscheidung über neu in den EBM aufzunehmende Leistungen bzw. GOPs und ihre Bewertung erfolgt im Regelfall durch den Bewertungsausschuss (BA), der sich aus von der KBV und dem GKV-Spitzenverband bestellten Vertretern zusammensetzt.

Soweit sich die Partner der Bundesmantelverträge (KBV und GKV-Spitzenverband) entsprechend verständigen können, besteht grundsätzlich auch die Möglichkeit, Leistungen und Leistungsbewertungen sowie weitere ergänzende Regelungen bundesmantelvertraglich $\mathrm{zu}$ vereinbaren. Eine Befassung im BA erübrigt sich in diesem Fall. Von einer Befassung im BA ausgenommen sind generell die angesprochenen Sachkostenpauschalen des Kapitels 40 des EBM, die auch nicht Gegenstand der Beratungen

3 Für einen Überblick: Telemedizinatlas im Auftrag der Bundesregierung, http://telemedizin.fokus.fraunhofer.de/ 
im BA sind, sondern die direkt zwischen den Partnern der Bundesmantelverträge vereinbart werden.

Von den Kostenpauschalen einmal abgesehen, ist für die Leistungsbeschreibungen im EBM charakteristisch, dass sie konsequent aus der Perspektive „ärztlichen Tuns“, also der konkreten ärztlichen Verrichtung bzw. Tätigkeit, beschrieben sind.

Übersicht 2 zeigt, wie die oben in Übersicht 1 ausgewiesene vorzufindende Heterogenität telemedizinischer Anwendungsmöglichkeiten mit im EBM in verschiedenen Konkretisierungen bereits beschriebenen ärztlichen Verrichtungen bzw. Tätigkeiten in einen Zusammenhang gesetzt werden kann.

Inwieweit spiegeln sich nun die Möglichkeiten, aktuell gültige EBM-Leistungen (rechte Spalte) telemedizinisch unterstützt zu erbringen (linke Spalte), in den konkreten EBM-Leistungsdefinitionen bzw. -beschreibungen?

Entgegen dem in unserem Alltag mittlerweile völlig selbstverständlichen Umgang mit moderner Kommunikationstechnologie ist der gegenwärtige EBM mit Blick auf die Erstellung von Arztbriefen, Berichten, Untersuchungsergebnissen und ihren Versand strukturkonservativ. Die Definition hier einschlägiger Kostenpauschalen, wie bspw. der Kostenpauschalen 40104, 40106, 40120 und 40122, stammt noch aus einer Zeit, in der Arztbriefe, Berichte und Befunde in Ermangelung anderweitiger Möglichkeiten mit der Post bzw. Boten befördert werden mussten. Eine Anpassung an die neuen Gegebenheiten ist bislang nicht erfolgt. Der EBM hinkt damit schon seit Jahren der Realität hinterher, in der Vertragsärzte sich wechselseitig Briefe, Berichte und Befunde auch elektronisch übermitteln. In welchem Umfang das der Fall ist, ist nicht bekannt, und nur am Rande sei angemerkt, dass auch nicht bekannt ist, inwieweit die aus datenschutzrechtlicher Sicht einzuhaltenden Sicherheitsvorkehrungen dabei beachtet werden.

Unter mittelbarem Arzt-PatientenKontakt bzw. Konsultation, angesprochen beispielsweise in der Leistungsbeschreibung zu GOP 01435, werden dem gängigen Verständnis folgend telefonische oder persönliche Kontakte insbesondere zu Angehörigen des Patienten verstanden, soweit diese der ärztlichen Betreuung des Patienten selbst dienen. Eine Online-Bildkonsultation zwischen Arzt und Patient wird darunter üblicherweise nicht verstanden und wird auch an keiner Stelle des EBM erwähnt (Primat des persönlichen Arzt-Patienten-Kontaktes).

Konsiliarische Befundbeurteilungen und konsiliarische Erörterungen/Beratungen sind als vom „Konsilgeber“ abrechnungsfähige Leistungen im EBM im Rahmen der schmerztherapeutischen Versorgung (GOP 30702), der Betreuung und Behandlung von Krebspatienten (GOP 07345 und weitere), der Beurteilung von Mammographieaufnahmen (GOP 01752) sowie als obligatorischer
Leistungsinhalt in der pädiatrisch-rheumatologischen Behandlung (GOP 04550) vorgesehen. Das Einholen eines Konsils dagegen ist definitionsgemäß eine sog. „Anhang 1-Leistung“ und ist als solche in der an mindestens einen persönlichen Arzt-Patienten-Kontakt gebundenen Versicherten- Grund- oder Konsiliarpauschale enthalten. Ein Hinweis oder eine Erläuterung mit Blick auf die bestehenden Möglichkeiten, ein Konsil auch basierend auf moderner Kommunikationstechnologie durchzuführen, findet sich im EBM nicht.

Die Teilnahme an multi- bzw. interdisziplinären Fallkonferenzen ist als EBMLeistung bzw. als Leistungsbestandteil einmal im Rahmen des Programms zur Früherkennung von Brustkrebs durch Mammographie-Screening vorgesehen (GOP 01758). In der Beschreibung zu GOP 01758 wird direkter Bezug auf die Mammographie-Richtlinie genommen. Hier findet sich in $\mathbb{S} 13$ Abs. 3 eine Regelung, nach der - soweit gemäß Anhang 13 Ziffer 2 eine entsprechende Genehmigung durch die KV vorliegt - die Fallkonferenz auch als Online-Bildkonferenz durchgeführt werden kann. Eine interdisziplinäre Fallkonferenz als EBMLeistung ist zum zweiten im Rahmen der schmerztherapeutischen Versorgung gemäß der Qualitätssicherungsvereinbarung zur schmerztherapeutischen Versorgung chronisch kranker Schmerzpatienten nach $\$ 135$ Abs. 2 SGB V vorgesehen (GOP 30706). GOP 30706 ist direkt auf

\section{Übersicht 2}

„Tele-“ Kurzbezeichnung

Gegenstand/Anwendungsfeld

Übermittlung von Arztbriefen und ärztlichen Berichten,

Telekommunikation ggf. unter Beifügung von Untersuchungsergebnissen (in einfachen Formaten wie pdf, gif, jpg)

Telekonsultation Online- bzw. Videokonsultation Konsultative Pathologie, konsultative und diagnostische Radiologie, konsultative und diagnostische Ophthalmologie

Telekonsil, Teleradiologie, Teleophthalmologie

Teleonkologie Fallkonferenzen Onkologie (Tumorkonferenzen)

Beobachtung und ggf. Anpassung der Therapie, insbe-

Telemonitoring, Telediabetes, Telekardiologie, Telepsychiatrie

Telepathologie

Telechirurgie sondere der medikamentösen Einstellung

Rund-um-die-Uhr-Überwachung von Vitalparametern bei Hochrisiko-patienten

Diagnostische Pathologie (zumeist bei Tumorverdacht) Chirurgische Eingriffe
„Passende“ Leistungs-beschreibung in der Diktion aktuell gültiger EBM-GOPs

Erstellung von Arztbriefen, ärztlichen Berichten, Untersuchungsergebnissen und Versand

Mittelbarer Arzt-Patienten-Kontakt

Konsiliarische Befundbeurteilung, konsiliarische Erörterung/Beratung

Teilnahme an multi-/interdisziplinären Fallkonferenzen

Verlaufsbeobachtung zur Therapiesicherung

Verlaufsüberwachung zur Notfallintervention

Untersuchung körpereigenen Materials Durchführung von Eingriffen 
\5 Abs. 3 dieser Qualitätssicherungsvereinbarung bezogen. Eine explizite Regelung zur Durchführungsmöglichkeit auch als Online-Bildkonferenz besteht hier nicht. Allerdings dürfte das auch praktisch schwierig werden, da die Anwesenheit des Patienten explizit gefordert wird.

In der Verlaufsbeobachtung zur Therapiesicherung werden aktuell im Rahmen von telemedizinischen Projekten Vitalparameter von Patienten über entsprechende Devices erfasst und elektronisch direkt in die Arztpraxis übermittelt. Dort werden sie vom Arzt im Hinblick auf ggf. erforderliche Therapieanpassungen gesichtet. Dafür finden sich mit einer Ausnahme gegenwärtig keine unmittelbar passenden GOPs im EBM. Diese Ausnahme bildet GOP 13552 „Funktionsanalyse eines Herzschrittmachers und/oder eines implantierten Kardioverters bzw. Defibrillators", die gemäß Leistungslegende ,auch mittels telemetrischer Abfrage" durchgeführt werden kann.

GOPs, die sich auf die Verlaufsüberwachung zur Notfallintervention gestützt auf via DFÜ online übermittelte Messwerte beziehen, finden sich dagegen gegenwärtig nicht im EBM. Warum das so ist und welche Konsequenzen daraus abgeleitet werden können, wird ausführlicher in Kapitel 4.3 behandelt.

Die beiden letzten „Typen“ prinzipiell telemedizinisch unterstützbarer ärztlicher Verrichtungen/Tätigkeiten aus Übersicht 1 und 2 werden im Weiteren ausgeblendet. Sowohl die „Untersuchung körpereigenen Materials durch einen nicht vor Ort anwesenden Experten" als auch die „Durchführung von Eingriffen durch einen nicht vor Ort anwesenden Experten" erfolgen unter Einsatz von Telemedizin typischerweise durch einen nicht vor Ort anwesenden Spezialisten (Pathologe bzw. Chirurg). Sie erfordern deshalb den Einsatz von ferngesteuerter Robotertechnik mit dazugehöriger Computer- und Kommunikationstechnik. Beide haben gegenwärtig noch keinen den anderen Anwendungen vergleichbaren Stand erreicht und kommen für eine Umsetzung in der vertragsärztlichen Versorgung gegenwärtig nicht in Betracht.

Zusammenfassend kann festgehalten werden, dass die bestehenden Möglich- keiten, vertragsärztliche Tätigkeit durch moderne Kommunikationstechnologie zu unterstützen, den EBM mit Ausnahme von zwei fest umrissenen Anwendungsfällen noch nicht erreicht haben. Einen Zugang, die Unterstützung ärztlicher Tätigkeit durch die Möglichkeiten der Telemedizin im gegenwärtigen EBM zu verankern, bietet die in Übersicht 2 dargestellte Zuordnung. Ausgehend von dieser Zuordnung kann nachfolgend ein konkreter Vorschlag für eine Berücksichtigung von Telemedizin im EBM formuliert werden.

Bei diesem Vorschlag wird auch berücksichtigt, dass die Leistungserbringung und die Berechtigung zur Abrechnung im aktuellen EBM für viele EBM-Leistungen neben dem Fachkundenachweis oftmals an eine gesonderte Genehmigung durch die jeweils zustän-

\section{Die Möglichkeiten, die} vertragsärztliche Tätigkeit durch moderne Kommunikationstechnologien zu unterstützen, hat den EMB nahezu nicht erreicht.

dige Kassenärztliche Vereinigung (KV) gebunden ist, die sich ihrerseits zumeist aus andernorts definierten spezifischen Anforderungen an die Erbringung einer Leistung ableitet. Das sind insbesondere Anforderungen gemäß Richtlinien des Gemeinsamen Bundesausschusses (G-BA) und Qualitätssicherungsvereinbarungen gemäß $\$ 135$ Abs. 2 SGB V. Richtlinien und Qualitätssicherungsvereinbarungen bestehen zum Beispiel für Leistungen, die unter Einsatz spezieller medizintechnischer Geräte erbracht werden oder bei denen der Einhaltung von Struktur- oder Prozessvorgaben aus qualitativen Gründen hohe Bedeutung beigemessen wird. Aktuell gilt das bereits für die bislang beiden einzigen im EBM explizit berücksichtigten Anwendungsfelder telemedizinisch unterstützter Leistungserbringung: Sowohl die Abrechnung der GOP 01758 als auch der GOP 13552 setzt eine Genehmigung durch die KV voraus. Erstere ausgehend von den Bestimmungen der MammographieRichtlinie, letztere ausgehend von der Vereinbarung zur Herzschrittmacherkontrolle gemäß \$135 Abs. 2 SGB V.

\section{Vorschlag zur Berücksichtigung von Telemedizin im EBM}

\subsection{Bestandteile und Überblick}

Der Vorschlag für die Berücksichtigung von Telemedizin im EBM setzt sich aus vier Bestandteilen zusammen:

1. Berücksichtigung der spezifischen Grundkosten einer Arztpraxis im EBM, die entsprechend den bestehenden Anforderungen an einen gesicherten Datenverkehr für den elektronischen Austausch patientenbezogener Informationen ausgerüstet ist (eArztpraxis)

2. Erweiterungen des im EBM berücksichtigten Leistungsspektrums

3. Vereinbarung der Partner der Bundesmantelverträge zu den technischen und organisatorischen Anforderungen an die telemedizinisch unterstützte Leistungserbringung in der Arztpraxis

4. Rahmensetzung für die Durchführung der Verlaufsüberwachung zur Notfallintervention (als zuerst zu schaffende Voraussetzung für eine Berücksichtigung im EBM)

Den Vorschlag für die Berücksichtigung telemedizinisch unterstützt erbrachter ärztlicher Leistungen im EBM entlang der oben vorgenommenen Zuordnungen (siehe oben Übersicht 2) zeigt nachstehende Abbildung 1.

Die vier Bestandteile werden nachfolgend detailliert beschrieben (Abschnitte 4.2 bis 4.5). Außerdem wird auf Aspekte der Finanzierung eingegangen (Abschnitt 4.6).

\subsection{Berücksichtigung der spezifischen Grundkosten einer „eArztpraxis“ im EBM}

Die telemedizinisch unterstützte ärztliche Leistungserbringung setzt Ausgaben für spezifische Anschaffungen und Installationen voraus (Hard- und Software, Router). Außerdem entstehen unabhängig von der Häufigkeit der Nutzung kontinuierlich Kosten für den laufenden Betrieb ${ }^{4}$. Aus

4 Neben der "Gebühr" für die Nutzung des Internets sind das insbesondere Kosten für die Sicherheitsvorkehrungen, die aufgrund der besonderen Schutzwürdigkeit patientenbezogener Informationen bzw. Daten erforderlich sind. In Abhängigkeit vom konkreten Anwendungskontext können das außerdem Kosten für höhere Servicelevel sein, wie sie aktuell Business-Tarife bieten. 


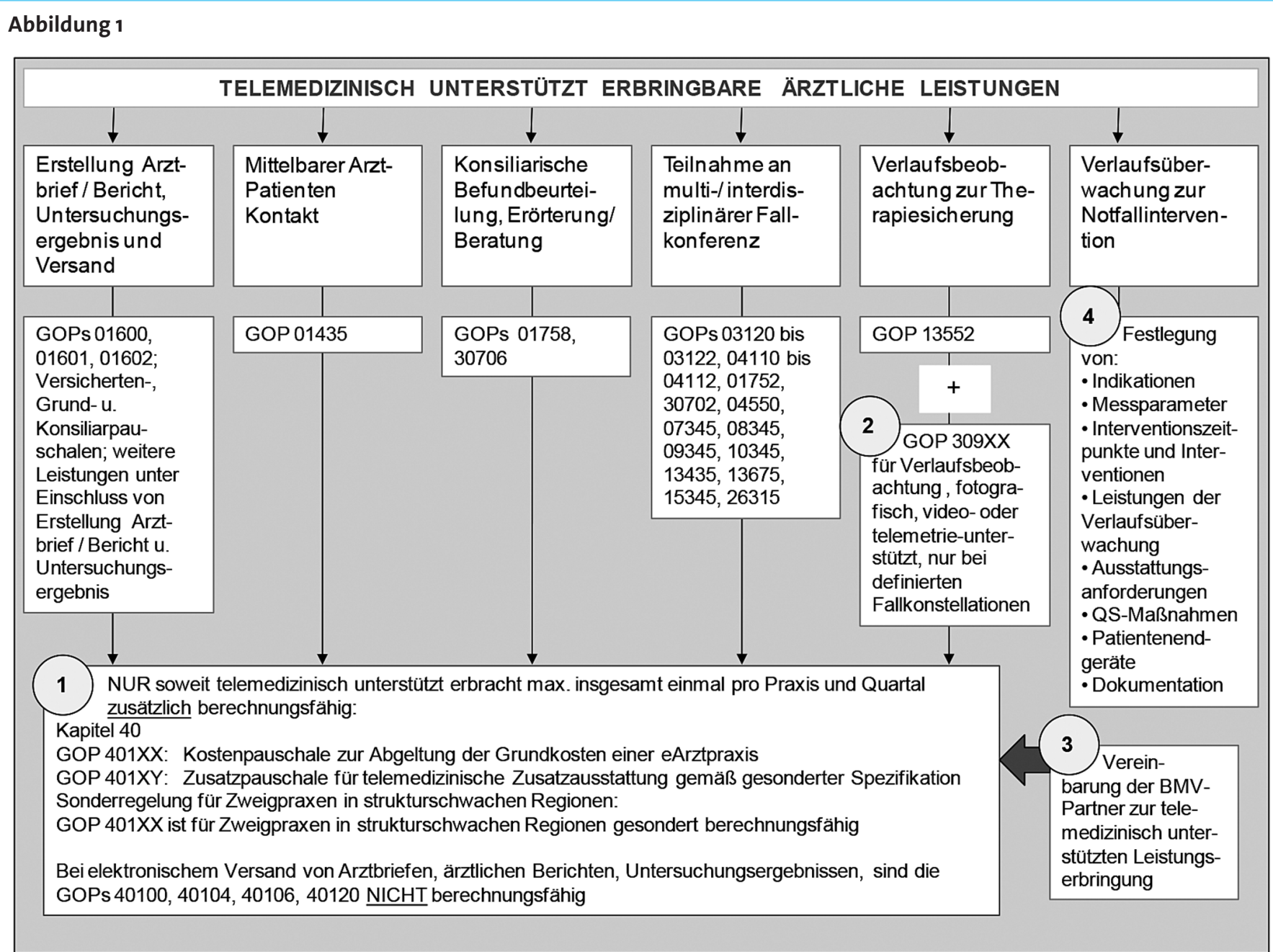

Quelle: AGENON Berlin

Sicht einer Praxis stellen diese Kosten zu den sonstigen Praxis-Fixkosten zusätzliche Fixkosten dar. Sie müssen von einer eArztpraxis unabhängig von der konkreten Nutzung im Einzelfall als Grundvoraussetzung dafür aufgebracht werden, dass ärztliche Tätigkeit überhaupt telemedizinisch unterstützt erbracht werden.

Im Grundsatz können davon die variablen Kosten unterschieden werden. Sie entstehen im Wesentlichen als Kosten zusätzlicher Arbeitszeit, soweit sich aus der Spezifik nunmehr telemedizinisch unterstützter Erbringung zusätzlicher zeitlicher Aufwand ergibt ${ }^{5}$.

Für eine Berücksichtigung der zusätzlichen Fixkosten für die telemedizinische Infrastruktur im EBM und ggf. bislang noch nicht berücksichtigte variable Kosten im genannten Sinne bestehen grundsätzlich zwei Zugangsmöglichkeiten:

- Die zusätzlichen Fixkosten werden über eine entsprechende Fixkostenpauschale in Kapitel 40 des EBM abgegolten. Die Abrechnung dieser Fix- kostenpauschale durch Vertragsärzte wird an die Voraussetzung geknüpft, dass die für eine telemedizinisch unterstützte Leistungserbringung erforderliche Infrastruktur tatsächlich auch zweckentsprechend genutzt wird. Praktisch wird dies umgesetzt, indem ihre Abrechnung an die Abrechnung telemedizinisch unterstützt erbrachter EBM-Leistungen gebunden wird.

- Die Abrechnung der Fixkostenpauschale wird zudem an die Voraussetzung gebunden, dass die von den Partnern der Bundesmantelverträge noch $\mathrm{zu}$ vereinbarenden technischen und organisatorischen Anforderungen an die telemedizinisch unterstützte Leistungserbringung in der Arztpraxis eingehalten werden (siehe dazu unten, Abschnitt 4.4). Ergänzend kann wie das in vielen anderen Fällen im EBM auch definiert ist - ein Nachweiserfordernis gegenüber der KV und eine Genehmigung zur Abrechnung der Fixkostenpauschale durch die KV vorgesehen werden.
- Soweit zusätzliche variable Kosten entstehen, die von der Bewertung der gegenwärtigen GOPs nicht abgedeckt sind, wird dies entsprechend der Struktur und der Systematik des EBM in den jeweiligen Kapiteln des EBM berücksichtigt. Soweit über den Einsatz der telemedizinisch-technischen Ausstattung weitere ärztliche Leistungen ermöglicht werden sollen, die über die bestehenden GOPs in medizinischer Hinsicht nicht

5 In vielen Fällen wird das für bereits im EBM berücksichtigte Leistungen nach unseren Abschätzungen gegenüber der konventionellen Leistungserbringung allerdings nicht der Fall sein. Bspw. dürfte der Ausdruck und postalische Versand eines Briefes oder ärztlichen Berichtes unter Beifügung eines Röntgenbildes in etwa mit dem gleichen zeitlichen Aufwand verbunden sein, der sich bei Zusammenstellung und Versand dieser Unterlagen in digitaler und entsprechend gesicherter Form ergibt. Im Rahmen der Verlaufsbeobachtung zur Therapiesicherung können sich demgegenüber durch die Nutzung der telemedizinischen Möglichkeiten auch Effizienzvorteile bei einer eArztpraxis ergeben, da der Patient zur Überwachung der Vitalparameter nicht extra einbestellt werden muss. 
abgebildet werden können, kann dies wiederum unter Berücksichtigung von Struktur und Systematik des EBM über Neudefinitionen berücksichtigt werden. Die Bewertung dieser Neudefinitionen erfolgt dann auf Ansätzen für die variablen Kosten und die sonstigen Praxis-Fixkosten.

- Zusätzliche fixe und variable Kosten werden in den Bewertungen der gegenwärtigen EBM-Ziffern, die für telemedizinisch unterstützt erbrachte Leistungen relevant sind, berücksichtigt. Soweit über den Einsatz der telemedizinisch-technischen Ausstattung weitere ärztliche Leistungen ermöglicht werden sollen, die über die bestehenden EBM-Ziffern in medizinischer Hinsicht nicht adäquat abgebildet werden können, kann dies wie oben - unter Berücksichtigung von Struktur und Systematik des EBM über Neudefinitionen berücksichtigt werden. Im Unterschied zu der im ersten Spiegelpunkt genannten Möglichkeit erfolgt die Bewertung aber auf Ansätzen sowohl für die variablen Kosten, die sonstigen PraxisFixkosten $u$ nd die zusätzlichen fixen Kosten. Alle anderen zuvor erfolgten Konkretisierungen gelten hier analog.

Die zuletzt genannte Möglichkeit bedingt, dass es auf Praxisebene sowohl zu einer Kostenunter- als auch zu einer Kostenüberdeckung kommen kann (vgl. Abbildung 2).

Dies wird der Fall sein, wenn die für die Umlage der Fixkosten notwendigerweise anzunehmenden Leistungshäufigkeiten (Menge $\mathrm{x}$ in Abbildung 2) falsch eingeschätzt werden bzw. wenn in einer Arztpraxis deutlich weniger oder mehr dieser Leistungen erbracht werden. Damit setzt diese zweite Variante auch Anreize entweder dazu, die Leistungen erst gar nicht telemedizinisch unterstützt $\mathrm{zu}$ erbringen, oder wenn, dann sehr extensiv.

Unser Vorschlag favorisiert deshalb die zuerst genannte Variante einer Fixkostenpauschale. Durch sie werden die Kosten für die erforderliche technische Infrastruktur dann abgegolten, wenn diese Infrastruktur auch tatsächlich zweckentsprechend genutzt wird.

Die Fixkostenpauschale kann maximal einmal pro Praxis und Quartal abgerechnet werden. Für die Verbesserung der Versorgung in strukturschwachen Regionen wird eine Ausnahmeregelung vorgesehen, nach der - jeweils gemäß

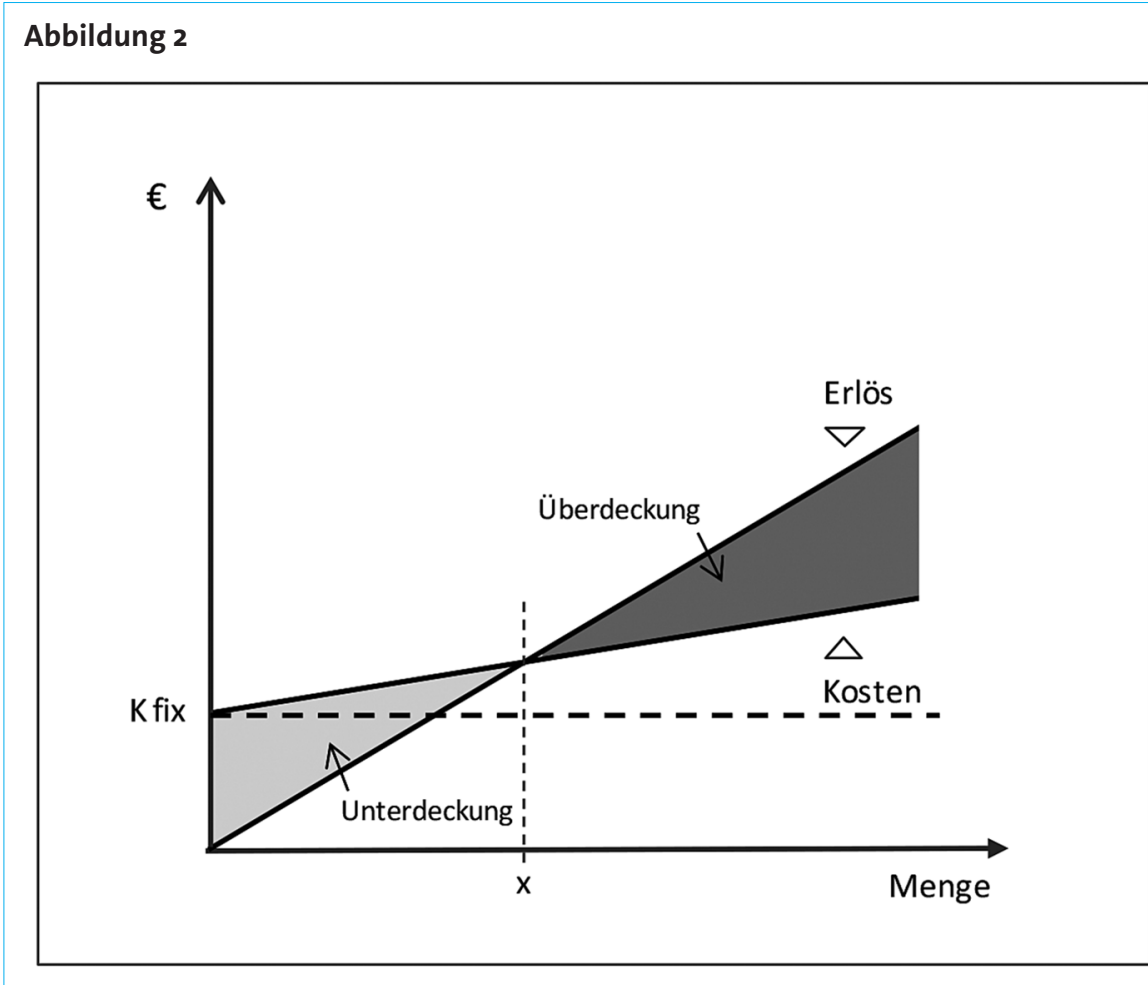

Quelle: AGENON Berlin

näherer Bestimmung durch die KV - die Fixkostenpauschale auch für eine Zweigpraxis in Ansatz gebracht werden kann.

Nach unseren Kostenberechnungen ergeben sich hier - je nach Sicherheitsund je nach Servicelevel -auf ein Quartal bezogene Kosten im Minimum von $152 €$ (niedrigstes Sicherheitslevel, ohne Kosten für Hard- und Software) und im Maximum von $440 €$ (höchstes Sicherheits- und Servicelevel, unter Berücksichtigung der Kosten für Hard- und Software). Definitionen und Kalkulationen sind ausgehend von Empfehlungen der KBV und der Bundesärztekammer „zur ärztlichen Schweigepflicht und Datenverarbeitung in der Arztpraxis“ vorgenommen worden ${ }^{6}$. Seitens der KBV werden diese Empfehlungen derzeit aktualisiert. Gegebenenfalls müssen Definitionen und Kalkulationen an den neuen Stand angepasst werden.

Es sind außerdem verschiedene Konstellationen denkbar, in denen die Fixkostenpauschale nicht ausreicht, um den aus versorgungspolitischer Sicht erwünschten Anforderungen nachzukommen. Das kann der Fall sein,

- wenn der Datendurchsatz bei der Datenübertragung so hoch ist bzw. die Übertragung spezieller Formate geleistet werden muss, so dass höhere (und teurere) Service-Level erforderlich sind (wenn zum Beispiel Be- fundbeurteilungen und die fachliche Erörterung/Beratung voraussetzen, dass 3D-Formate übertragen werden); - wenn die ärztliche Beobachtung bzw. Überwachung von Vitalparametern eine (komplexere) Vorverarbeitung eingehender Daten voraussetzt, für die spezielle Software angeschafft werden muss.

Konstellationen dieser Art können in Kapitel 40 über Zusatzpauschalen zur Fixkostenpauschale für die telemedizinischen Vorhaltungen abgebildet werden.

\subsection{Erweiterungen des im EBM berücksichtigten Leistungsspektrums}

Es wurde gezeigt (oben Kapitel 3), dass die telemedizinische Unterstützung ärztlicher Tätigkeit bislang nur in einigen wenigen und fest umrissenen Anwendungsfällen explizit im EBM berücksichtigt ist. Damit haben viele bestehende Möglichkeiten, die ärztliche Tätigkeit telemedizinisch zu unterstützen, (noch) keinen Niederschlag im EBM gefunden.

6 Vgl. Bundesärztekammer und Kassenärztliche Bundesvereinigung (2008), Empfehlungen zur ärztlichen Schweigepflicht und Datenverarbeitung in der Arztpraxis, in: Deutsches Ärzteblatt, Jg. 105, Heft 19: A1026 - A1030. 
Dazu gehören bspw. Online-Konsile zwischen Pathologen und interdisziplinäre Online-Fallkonferenzen auch für Indikationen jenseits der Diagnostik und Therapieabstimmung im Rahmen der Behandlung von Krebserkrankungen.

Es würde an dieser Stelle zu weit führen, hier einen entsprechend aufgefächerten Vorschlag für Möglichkeiten zur Berücksichtigung im EBM zu formulieren, zumal dahingehend „öffnende“ Vereinbarungen, vor allem in den Bundesmantelverträgen oder über Qualitätssicherungsvereinbarungen gemäß $\ 135$ Abs. 2 SGB V, bislang ebenso fehlen wie Richtlinien des G-BA.

Beispielhaft wird nachfolgend zumindest aber für die telemedizinisch unterstützte Verlaufsbeobachtung zur Therapiesicherung ein konkreter Vorschlag unterbreitet. Sie wurde und wird in Projekten schon seit Jahren praktiziert, zum Beispiel zur medikamentösen Einstellung von Diabetikern (metabolischer Fingerabdruck), von Patienten mit Asthma bronchiale/COPD, von ParkinsonPatienten und Patienten mit Multipler Sklerose. Der bislang einzige im EBM berücksichtigte Anwendungskontext „Funktionsanalyse eines Herzschrittmachers und/oder eines implantierten Kardioverters bzw. Defibrillators, (...) auch mittels telemetrischer Abfrage“ (GOP 13552) bleibt damit weit hinter den Möglichkeiten zurück, die hier heute bestehen. Ihnen könnte etwa durch die Einführung einer bzw. je nach medizinischem Anwendungskontext verschieden gestalteter GOPs Rechnung getragen werden.

Ein konkreter Vorschlag im Fall nur einer GOP wäre eine neue GOP 309XX, die in einem (ebenfalls neuen) Kapitel 30.11 - Verlaufsbeobachtung des EBM wie folgt konkretisiert wird:

Verlaufsbeobachtung zur Therapiesicherung, insbesondere der medikamentösen Einstellung, fotografisch, video-oder telemetrie-unterstützt. GOP 309XX ist maximal dreimal pro Behandlungsfall berechnungsfähig.

GOP 309XX wird außerdem in den Ziffernkranz aufgenommen, der zur Abrechnung der oben vorgeschlagenen Fixkostenpauschale für die technische Infrastruktur berechtigt, die für eine telemedizinisch unterstützte Leistungserbringung erforderlich ist.

\subsection{Vereinbarung zu den technischen und organisa- torischen Anforderungen}

Es kann davon ausgegangen werden, dass eine breitere Nutzung der Möglichkeiten, die der elektronisch gestützte Austausch von patientenbezogenen Informationen in der ambulanten ärztlichen Versorgung grundsätzlich ermöglicht, auch durch Unsicherheiten auf der Anwenderseite den in freier Praxis oder angestellt ambulant tätigen Ärzten - gehemmt wird. Die Unsicherheiten rühren oftmals noch aus der Vergangenheit, in der vor einer Anbindung der elektronischen Praxisverwaltungssysteme an das Internet eindringlich gewarnt wurde. Als Beitrag zur Überwindung dieser Hemmnisse wird vorgeschlagen, dass sich die Partner der Bundesmantelverträge auf technische und organisatorische Voraussetzungen für die telemedizinisch unterstützte Leistungserbringung in der Arztpraxis verständigen und entsprechende Anforderungen vereinbaren (Vereinbarung als Bestandteil der Bundesmantelverträge oder Qualitätssicherungsvereinbarung gemäß \135 Abs. 2 SGB V).

Die Grundausstattung für die Telemedizin ist unabhängig von dem Aufbau der zukünftigen Telematik-Infrastruktur zu sehen. Hierbei handelt es sich aus unserer Sicht um einen abgesetzten Rechner, versehen mit Software und User-Interface für einen telemedizinischen Arbeitsplatz und den branchenspezifischen Anforderungen entsprechenden Datensicherheits- und schutzvorkehrungen, beispielsweise auch abgesicherter Videokommunikation.

Eine solche Grundausstattung wird durch die Telematik-Infrastruktur nicht überflüssig. Im Gegenteil: Sie kann wie andere Technologiekomponenten (z.B. KV Safenet) ohne größere Aufwände in die zukünftige Basisinfrastruktur integriert werden. Eine zügige Verbreitung von Telemedizin würde damit auch dafür sorgen, dass die Anwendungen für die Telematik-Infrastruktur rascher zur Verfügung stehen und damit die Infrastruktur früher und besser ausgelastet wird.

Während die telemedizinische Infrastruktur dafür sorgt, dass der Arzt die medizinische Versorgung von Patienten, telemedizinisch unterstützt durchführen kann, ist es Aufgabe der Telematik-Infrastruktur, zwischen den professionellen Akteuren Datenaustausch und elektronische Kommunikation standardisiert und strukturiert bereitzustellen. Mit anderen Worten: Auch bei ausgerollter TelematikInfrastruktur wird es eine davon separate telemedizinische Grundausstattung geben. Auch eine telemedizinische Basistechnologie bedarf mittelfristig mithin der Ergänzung um eine Telematik-Infrastruktur.

\subsection{Rahmensetzungen für die Verlaufsüberwachung zur Notfallintervention}

Bei der Verlaufsbeobachtung zur Therapiesicherung können eingehende $\mathrm{Vi}$ talparameter vom Arzt auch zeitversetzt gesichtet und bewertet werden. Bei der Verlaufsüberwachung zur Notfallintervention, beispielsweise bei Hochrisikopatienten mit Herzinsuffizienz oder mit Diabetes mellitus, ist es dagegen erforderlich, dass Vitalparameter praktisch zeitgleich gesichtet und bewertet werden. Das lässt sich in einer „normalen" Praxis nur schwerlich im laufenden Praxisbetrieb bewerkstelligen. Ferner muss gesichert sein, dass die mitlaufende Sichtung und Bewertung auch jenseits der Praxisöffnungszeiten erfolgt und im Notfall die dafür vorgesehenen Maßnahmen eingeleitet und umgesetzt werden. Dafür ist ständig anwesendes spezialisiertes Fachpersonal erforderlich. Außerdem muss das technische Equipment, das für eine effektive Online-Überwachung bis hin zur Notfallintervention erforderlich ist, angeschafft, betrieben und gewartet werden. Daraus resultieren Kosten und Kostenstrukturen, die sich deutlich von denen „normaler“ Vertragsarztpraxen unterscheiden. Für einen wirtschaftlichen Betrieb sind zudem Skaleneffekte erforderlich, die sich nur mit einer großen Anzahl überwachter Patienten erreichen lassen werden. Schließlich wird sich das Leistungsspektrum der Verlaufsüberwachung zur Notfallintervention von dem Leistungsspektrum von Vertragsarztpraxen, deren Kosten und Kostenstrukturen üblicherweise zur Bewertung von EBM-Leistungen (über das sogenannte STABS) herangezogen werden, deutlich unterscheiden. Nicht ohne Grund werden derartige Leistungen im Rahmen von Projekten gegenwärtig entweder durch an Krankenhäuser angesiedelten oder durch entsprechend spezialisierte und ausgerichtete solitäre Telemedizinische Zentren erbracht. 
Für eine regelhafte Vergütungsmöglichkeit über den EBM fehlen überdies bislang Regelungen und Festlegungen, wie sie ansonsten für entsprechend spezialisierte Leistungen bestehen, die maßgeblich gestützt auf moderne Technologie erbracht werden. Insofern ist gegenwärtig festzustellen, dass die Voraussetzungen für die Formulierung von EBM-GOPs und ihre Bewertung in mehreren Hinsichten noch nicht gegeben sind.

$\mathrm{Zu}$ regeln wären insbesondere

- Indikationen und Einschlusskriterien, bei denen eine Verlaufsüberwachung zur Notfallintervention zu Lasten der Krankenkassen erbracht werden kann, und Ausschlusskriterien, bei denen dies nicht zulässig ist;

- Parameter, die zur Verlaufsüberwachung notwendig und zu übertragen sind;

- Interventionszeitpunkte und Interventionen;

- Leistungen und Angaben zur Dauer der zu Lasten der Krankenkassen finanzierbaren Verlaufsüberwachung;

- Ausstattungsanforderungen in räumlicher, technischer, sachlicher und personeller Hinsicht sowie Standards und Anforderungen, die mindestens einzuhalten sind;

- Art und Anforderungen an Endgeräte beim Patienten, einzuhaltende Standards und Anforderungen;

- Maßnahmen zur Qualitätssicherung und Dokumentationserfordernisse.

Der Regelungsbedarf kann prinzipiell unterschiedlich abgedeckt werden: Über bundesmantelvertragliche Vereinbarungen, über Qualitätssicherungsvereinbarungen gemäß $\$ 135$ Abs. 2 SGB V, und schließlich können entsprechende Regelungen auch durch den G-BA erfolgen, sofern er damit beauftragt wird.

\subsection{Zur Frage der Finanzierung}

Zur Frage der Finanzierung der neuen Positionen im EBM: Der mit dem oben skizzierten Vorschlag verbundene Fluss von finanziellen Mitteln kann prinzipiell entweder zusätzlich von den gesetzlichen Krankenkassen und damit letzten Endes den GKV-Beitragszahlern - aufgebracht werden, oder die Finanzierung kann aus den ansonsten zur Verfügung stehenden Mitteln erfolgen. Die Klärung dieser Frage erfolgt über
Vereinbarungen zwischen Arzt- und Kassenseite.

Zur Finanzierung von Endgeräten beim Patienten: Soweit die telemedizinisch unterstützte Leistungserbringung bedingt, dass Informationen vom Patienten elektronisch übermittelt werden, setzt das entsprechende Patientenendgeräte voraus: Im Rahmen der Verlaufsbeobachtung zur Therapieanpassung bei Diabetes-Patienten beispielsweise ein Glukose-Messgerät und ein Modem, für die Überwachung zur Notfallintervention bei Herzinsuffizienzpatienten beispielsweise eine elektronische Waage, ein Blutdruckmessgerät und ein Modem.Eine Tragung der damit verbundenen Kosten im Rahmen der gesetzlichen Krankenversicherung kann über eine Aufnahme in den Hilfsmittelkatalog der GKV erfolgen. Diskutiert wird als zweite Variante, die Beschaffung, die Vornahme der gegebenenfalls erforderlichen Installationen und die Wartung als Teil der vertragsärztlichen Leistung auszugestalten. Das hätte zur Konsequenz, dass der Arzt sich entsprechende Leistungspakete über Verträge bei geeigneten Anbietern selbst „einkaufen“ müsste.

\section{Ausblick}

Abgestimmt auf die gegenwärtige Struktur des EBM wurden für die Berücksichtigung von Telemedizin im EBM konkrete Vorschläge unterbreitet. Es wurde außerdem vorgeschlagen, dass die Partner der Bundesmantelverträge mit einer Vereinbarung zu den technischen und organisatorischen Anforderungen an die telemedizinisch unterstützte Leistungserbringung in der Arztpraxis bestehende Hemmnisse auf der Anwenderseite beseitigen. Um die Voraussetzungen für eine Vergütungsmöglichkeit der Leistungen Telemedizinischer Zentren über den EBM zu schaffen, wurden außerdem Felder benannt, für die dazu noch weiterer Regelungsbedarf besteht. Damit steht ein konkreter Vorschlag zur Diskussion, über den die gesetzlichen Aufträge formuliert in den $\mathbb{S} \int 67$ und 87 Abs. 2a Satz 8 SGB V - umgesetzt werden können.

Die Vorschläge berücksichtigen, dass viele Ärzte und Ärzteorganisationen telemedizinischen Ansätzen auch deshalb skeptisch gegenüber stehen, weil in den derzeit laufenden Projekten oftmals der Dialog mit dem Patienten fast vollständig durch den Telemedizinanbieter substituiert wird. Der niedergelassene Arzt findet sich in der Rolle des Informationsnehmers in Form eines vierteljährlichen Arztbriefes aus einem TMZ wieder.

Wird die telemedizinisch unterstützte medizinische Versorgung entlang der hier formulierten Vorschläge weiter entwickelt, so wird auch die Integration mit den als Bestandteil der GKV-Regelversorgung spätestens für die Verlaufsüberwachung zur Notfallintervention erforderlichen TMZ vereinfacht.

Soweit zudem die mittlerweile etablierten elektronischen Kommunikationswege zwischen Vertragsarzt und KV genutzt werden können, würde die Definition neuer Schnittstellen entfallen und damit auch eine Quelle lähmender Verhandlungsprozesse zwischen Arzt- und Industrieseite.

Die Organisation telemedizinisch unterstützter ambulanter ärztlicher Versorgung als Bestandteil der GKV-Regelversorgung überführt diese von Managementgesellschaften und klinischer Forschung wesentlich in die Obhut des niedergelassenen Arztes. Durch die Integration in die Arztpraxis werden Telemedizin-Provider gezwungen, ihre Services so zu entwickeln, dass sie in die tägliche Versorgungspraxis und vor allem in die Praxisverwaltungssysteme - möglichst reibungslos technisch integriert werden können und von den Patienten möglichst einfach genutzt werden. Das sind exakt die richtigen Anreize, um telemedizinische Lösungen nachhaltig zu einem festen Bestandteil der ambulanten ärztlichen GKV-Versorgung werden zu lassen.

Auf diese Weise wird auch ein Prozess in Gang gesetzt, der helfen wird, das heutige Dilemma fast 300 notierter, nicht miteinander vernetzter Telemedizinprojekte in Deutschland zu überwinden. Diese Projekte sind heute ganz überwiegend „Arzt-fern“ mit separater Infrastruktur aufgebaut worden. Nach Projekt- bzw. Förderende können diese Projekte im Regelfall nicht weitergeführt werden, weil sie als Projekt und nicht als Teil einer größeren, flächendeckenden Infrastruktur entwickelt worden sind. Ein arztzentriertes Telemedizinmodell würde aber genau diese technologische Entwicklung fördern: Das Entstehen einer bundesweiten Telemedizin-Infrastruktur, die dann zu deutlich verringerten Zusatzkosten für die telemedizinisch unterstützte Regelversorgung, nach wie vor aber auch für selektivvertragliche Versorgungsansätze genutzt werden kann. 